\title{
Editorial
}

\section{PTEN, here, there, everywhere}

\author{
C Bassi ${ }^{1}$ and V Stambolic ${ }^{\star, 1,2}$ \\ Cell Death and Differentiation (2013) 20, 1595-1596; doi:10.1038/cdd.2013.127
}

Loss of function of the phosphatase and tensin homolog (PTEN) tumor suppressor is frequently found in many human malignancies. ${ }^{1}$ PTEN antagonizes the phosphatidylinositide 3-kinase (PI3K) pathway ${ }^{2}$ by dephosphorylating the $3^{\prime}$ position of phosphatidylinositol $(3,4,5)$-trisphosphate $\left[\right.$ Ptdlns $\left.(3,4,5) \mathrm{P}_{3}\right]$ (or PIP3). ${ }^{2,3}$ PIP3 serves as a second messenger whose levels in the plasma membrane are elevated following cell stimulation with growth factors, mediated by the activity of PI3Ks. Proteins containing pleckstrin homology $(\mathrm{PH})$ domains physically interact with PIP3, bringing them into close proximity with other $\mathrm{PH}$ domain-containing proteins and facilitating further functional interactions that serve to propagate the membranous signal. ${ }^{4}$

PTEN contains an N-terminal phosphatase domain that displays activity not only toward phosphatydilinositol, lipid substrates, but also toward proteinacious ones, although the search for physiologically relevant protein PTEN substrates continues. ${ }^{5}$ The C-terminal half of PTEN features a $\mathrm{Ca}^{2+}$. independent $\mathrm{C} 2$ domain thought to mediate PTEN interactions with the plasma membrane. ${ }^{6}$ A cluster of cationic residues of the $\beta$-sandwich, composed of eight $\beta$-strands, on the membrane-binding face of PTEN appear to mediate membrane anchoring. ${ }^{6}$ Recent evidence suggests further complexity of these interactions, namely, that PTEN SUMOylation at $\mathrm{K}^{266}$ located within the CBR3 loop has a central role in PTEN membrane association, facilitating the binding of PTEN to the plasma membrane via electrostatic interactions. ${ }^{7}$ However, structural analysis using neutron reflectometry challenges this model and demonstrates that the CBR3 loop of PTEN's C2 domain, as well as further electrostatic interactions of the phosphatase domain, is sufficient for membrane association, independent of SUMOylation. ${ }^{8}$ PTEN unstructured $\mathrm{C}$ terminus, consisting of the last 50 amino acids, has also been implicated in PTEN membrane localization. Guanylate kinase with inverted orientation (MAGI) proteins, which contain PDZ domains, has been shown to bind to the PTEN C-terminal PDZ-domain interaction sequence and reinforce PTEN interaction with the plasma membrane..$^{9,10}$

In addition to membrane and cytoplasmic localization, which can be easily associated with its function in regulating the levels of $3^{\prime}$ phosphorylated phosphatidylinositols, a number of reports, including several recent ones discussed below, point to specific localization of PTEN to other cellular compartments, where it may exert other tumor suppressive

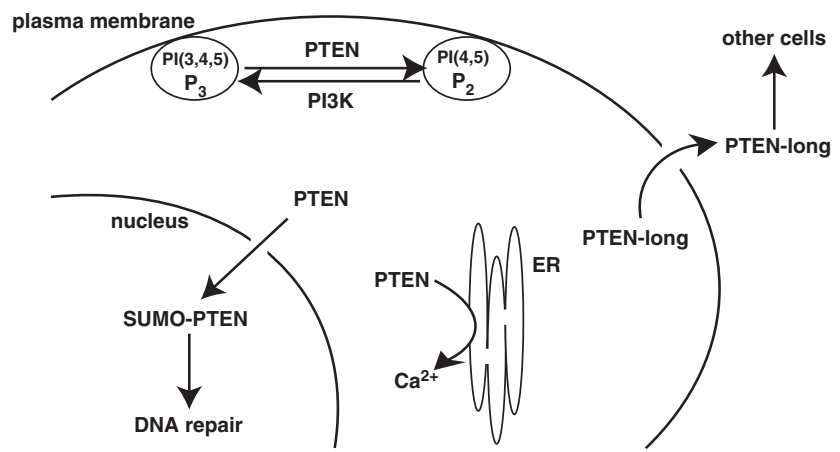

Figure 1 PTEN acts within several cellular compartments (see text for details)

functions (Figure 1). For instance, PTEN is readily found in the nuclei of many cultured cells and tissues, including normal breast epithelium, proliferating endometrium, normal pancreatic islet cells, vascular smooth muscle cells, follicular thyroid cells, squamous cell carcinoma and primary cutaneous melanoma. ${ }^{11}$ Although nuclear phosphatidylinositols have been reported, they are a part of distinct, partially detergent-resistant proteolipid complexes that are not dynamically regulated and are not likely PTEN substrates. ${ }^{12}$ Numerous molecular mechanisms responsible for PTEN nuclear localization have been proposed. These include the putative nuclear localization signals within PTEN that mediate its interaction with the major vault protein, ${ }^{11} \mathrm{~N}$-terminal sequences responsible for Ran-mediated nuclear transport ${ }^{11}$ and a potential PI3K signaling-sensitive, cell cycle-regulated PTEN nuclear export mechanism. ${ }^{11}$ Monoubiquitinationmediated PTEN nuclear localization has also been reported, ${ }^{13}$ although this mechanism of PTEN nuclear localization is not fully elucidated. ${ }^{14,15}$ PTEN may also have a cytoplasmretention/nuclear export sequence within its $\mathrm{N}$ terminus. ${ }^{11}$ Interestingly, mutations within this region of PTEN result in its constitutive nuclear localization, precluding its growthsuppressive function at the plasma membrane. ${ }^{11}$

Another recently discovered mechanism of PTEN nuclear localization involves SUMOylation-mediated PTEN nuclear retention. ${ }^{16}$ Interestingly, SUMOylated nuclear PTEN participates in the cellular response to DNA damage, helping to explain the genomic instability of PTEN-deficient tumors and their sensitivity to poly(ADP-ribose) polymerase inhibitors. ${ }^{17}$ This function of PTEN relates to its protein

${ }^{1}$ Department of Medical Biophysics, University of Toronto, Toronto, Ontario, Canada M5G 2M9 and ${ }^{2}$ Division of Signaling Biology, Princess Margaret Cancer Center, Ontario Cancer Institute/University Health Network, Toronto, Ontario, Canada M5G 2M9

${ }^{*}$ Corresponding author: V Stambolic, Division of Signaling Biology, Princess Margaret Cancer Center, Ontario Cancer Institute/University Health Network, 610 University Avenue, Toronto, Ontario, Canada M5G 2M9. Tel: +416 946 2961; Fax: +416 946 2985; E-mail: vuks@uhnres.utoronto.ca 
but not lipid phosphatase activity and is regulated by Ataxia telangiectasia mutated, a DNA damage-induced PIKK kinase. $^{16}$

Reporting recently in this journal, Bononi and co-workers ${ }^{18}$ showed that a fraction of PTEN localizes to the endoplasmatic reticulum (ER) and mitochondria-associated membranes. Here, PTEN appears to regulate the $\mathrm{Ca}^{2+}$ release from the $E R$ in a protein phosphatase-dependent manner that counters the $\mathrm{PKB} / \mathrm{Akt}$-mediated reduction of $\mathrm{Ca}^{2}+$ release via the inositol 1,4,5-trisphosphate receptors, with which PTEN surprisingly interacts. ${ }^{18}$ Reduction in the kinetics of $\mathrm{Ca}^{2+}$ release from the ER upon PTEN loss may contribute to reduced sensitivity to apoptosis. ${ }^{18}$ Finally, studying a PTEN protein initiated from an alternate translation start site, a secreted PTEN polypeptide has also been discovered. ${ }^{19}$ This fully phosphatase competent protein named PTEN-long appears capable of entering cells and regulating the PI3K signaling pathway in them. ${ }^{19}$

PTEN cellular distribution and delivery clearly represent key aspects of PTEN regulation, targeting PTEN phosphatase activity toward distinct pools of its substrates. Apart from the impact of PTEN phosphorylation on a cluster of C-terminal residues, which may inhibit $\mathrm{PTEN},{ }^{20}$ there is little evidence for mechanism(s) of dynamic regulation of PTEN lipid phosphatase activity. Even less is known about the control of PTEN protein phosphatase function, reflecting the need for a comprehensive assessment of PTEN protein phosphatase activity and systematic identification of PTEN protein substrates. Deeper understanding of the intricacy of PTEN control will offer an insight into the cellular landscape of PTEN activity and function, likely to involve several processes and targets. This expanded knowledge will certainly aid in the clinical management and treatment of human cancers when PTEN is lost or mutated.

\section{Conflict of Interest}

The authors declare no conflict of interest.

1. Li J et al. Science 1997; 275: 1943-1947.

2. Maehama T, Dixon JE. J Biol Chem 1998; 273: 13375-13378.

3. Stambolic V et al. Cell 1998; 95: 29-39.

4. Haslam RJ, Koide HB, Hemmings BA. Nature 1993; 363: 309-310.

5. Tamura M et al. J Biol Chem 1999; 274: 20693-20703.

6. Lee JO et al. Cell 1999; 99: 323-334.

7. Huang J et al. Nat Commun 2012; 3: 911.

8. Shenoy SS, Nanda H, Losche M. J Struct Biol 2012; 180: 394-408.

9. Wu X et al. Proc Natl Acad Sci USA 2000; 97: 4233-4238.

10. Wu $Y$ et al. J Biol Chem 2000; 275: 21477-21485.

11. Planchon SM, Waite KA, Eng C. J Cell Sci 2008; 121(Pt 3): 249-253.

12. Lindsay $Y$ et al. J Cell Sci 2006; 119(Pt 24): 5160-5168.

13. Trotman LC et al. Cell 2007; 128: 141-156.

14. Fouladkou F et al. Proc Natl Acad Sci USA 2008; 105: 8585-8590.

15. Bernassola F et al. Cancer Cell 2008; 14: 10-21.

16. Bassi C et al. Science 2013; 341: 395-399.

17. Mendes-Pereira AM et al. EMBO Mol Med 2009; 1: 315-322.

18. Bononi A et al. Cell Death Differ 2013; 20: 1631-1643.

19. Hopkins BD et al. 2013; 341: 399-402.

20. Vazquez F et al. Mol Cell Biol 2000; 20: 5010-5018. 